Conclusions Central neuraxial blocks could be alternative techniques for colorectal ESD procedures, especially for fragile patients [3]. Procedure duration could not be accurately predicted, thus continuous epidural or CSE, should be preferred. Research trials are needed to corroborate our thoughts.

\section{INCIDENCE AND MANAGEMENT OF POST-DURAL PUNCTURE HEADACHE AND ACCIDENTAL DURAL PUNCTURE FROM AN ONCOLOGY HOSPITAL: A 5-YEAR RETROSPECTIVE ANALYSIS}

S Dias, M Nunes Ferreira, J Oliveira*, S Serra, P Alves, M Gacio, L Miranda. Instituto Português de Oncologia do Porto, Porto, Portugal

\subsection{6/rapm-2021-ESRA.47}

Background and Aims Accidental dural puncture (ADP) and post-dural puncture headache (PDPH) are epidural anaesthesia's complications. There's limited evidence in non-obstetric patients and no consensus management.

The aim of this study was to evaluate its incidence and approach in an institution where combined epidural-general anaesthesia is preferential.

Methods Retrospective analysis (SPSS V.26) was conducted of adult patients submitted to elective surgery with combined epidural-general anaesthesia and suffered ADP, april 2015-2020. Information about the patient's background, epidural procedure, PDPH management and clinical evolution was collected from clinical records.

Results 3237 patients have had a combined epidural-general anaesthesia, 31 suffered ADP (0,96\%). 61,3\% were female, $71 \%$ ASA II, mean age 59,61 years. 6 patients developed $\mathrm{PDPH}, 1$ without previous ADP identification, resulting in an incidence of PDPH of 19,35\%. This incidence was not statically different in patients in whom the catheter was resited $(n=5)$ comparing to the ones that were not $(n=1)$. All patients were treated conservatively, although not uniformly. The onset of headache was on average $48 \mathrm{~h}(24-72 \mathrm{~h})$ postoperative and with an average duration of $48 \mathrm{~h}$ (24-96h). 5 patients were submitted to prophylactic treatment for PDPH and only 1 developed PDPH; comparing to 5 in 26 that did not receive prophylactic treatment, although not statistically different. This study was approved by the Ethics Committee.

Conclusions The incidence of ADP and PDPH were lower than that reported in literature. Conservative treatment for $\mathrm{PDPH}$ was enough to manage this condition efficiently. The approach is not uniform, emphasizing the need of clinical protocol.

\section{INTRAOPERATIVE INTRATHECAL MORPHINE FOR POSTOPERATIVE ANALGESIA IN OPEN ABDOMINAL HYSTERECTOMY FOR GYNECOLOGICAL MALIGNANCY}

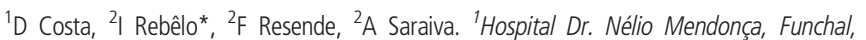
Portugal; ${ }^{2}$ Instituto Português de Oncologia de Lisboa Francisco Gentil, Lisboa, Portugal

\subsection{6/rapm-2021-ESRA.48}

Background and Aims Open abdominal hysterectomy is one of the most performed oncological gynecological surgeries, with an expected moderate to high pain level.
The use of single shot intrathecal morphine (ITM) has an analgesic effect of up to $24 \mathrm{~h}$, with patients requiring fewer postoperative IV opioids.

The aim of this study is comparing the analgesic effects and adverse effects of ITM against IV morphine, in cancer patients submitted to open abdominal hysterectomy.

Methods Prospective observational study.

Data collection over a 1-year period.

Inclusion criteria comprised adults submitted to total or radical open abdominal hysterectomy, with total intravenous anaesthesia with intraoperative propofol and fentanyl. Patients with pre-induction ITM administration (100 to $300 \mathrm{mcg})$ and postoperative analgesia with IV morphine patient controlled analgesia and paracetamol, were compared to a control group without spinal opioid.

Descriptive and comparative analysis for analgesic quality and adverse effects in the first 24 hour postoperative period was performed using SPSS software.

Results 36 patients were included in the ITM group and 44 in the control group. Comparative analysis found no significant association between ITM administration and lower static or dynamic pain scores. The ITM group had less PCA rescues $(\mathrm{p}<0.001)$ but a higher risk of postoperative vomiting $(22 \%$ vs $2.2 \%, \mathrm{p}<0.01)$.

Conclusions ITM administration resulted in significantly less PCA rescue, without perceived analgesic improvement compared with no ITM administration. Patients exhibited more side effects, such as nausea and vomit. Prescribing fixed antiemetics for the first 24 hours might be the best strategy to overcome these side effects.

\section{COMPARISON OF ANTI-SHIVERING EFFECT OF INTRAVENOUS VERSUS INTRATHECAL TRAMADOL IN PATIENTS UNDERGOING LOWER LIMB SURGERY UNDER SPINAL ANAESTHESIA}

EMohamed Abd El Azeem*, M Abdel-Ghaffar, SA Al-Touny. Suez Canal University, Ismailia, Egypt

\subsection{6/rapm-2021-ESRA.49}

Background and Aims Shivering occurs in 40-60 percent of patients under spinal anaesthesia. (1)

Prophylaxis with intravenous tramadol produces a dosedependent reduction in the incidence of shivering. (2) Tramadol is a common intrathecal adjuvant, safely used in a dose up to $20 \mathrm{mg}$. Few studies tested the anti-shivering efficacy of intrathecal tramadol. ${ }^{(3,4)}$ However, no study compared the anti-shivering effect of intravenous versus intrathecal tramadol. Therefore, we conceptualized this trial to compare the antishivering effect of intravenous versus intrathecal tramadol.

Methods This study was a randomized, double-blinded clinical trial that included 86 ASA I \& II patients divided into two equal groups. It included patients aged 18-65 years who undergone lower limb orthopedic surgeries; that lasted less than two hours under spinal anesthesia. Our institutional ethical committee approved the protocol and was registered at PACTR (trial ID: PACTR202007664590852). Patients were allocated randomly into control (IV) group and interventional (IT) group. The Control group received intrathecal $15 \mathrm{mg}$ bupivacaine then IV tramadol $25 \mathrm{mg}$ in $5 \mathrm{ml}$ normal saline. The interventional group received $20 \mathrm{mg}$ tramadol added to15 mg bupivacaine intrathecally then IV $5 \mathrm{ml}$ normal saline. 
The primary outcome was the incidence of intra-operative shivering which was assessed by Bedside Shivering Assessment Scale. ${ }^{(5)}$ Patients who developed intraoperative shivering were given $25 \mathrm{mg}$ IV tramadol.

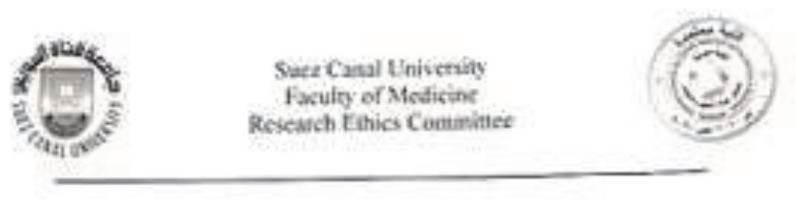

Dater 10sacos

Dri Einas Statimed Abd E Areem

Depariment: Anentesir ond Critical Cuse, Faculy of Medicine, Susz Canal Laiversity liaspios

Referencti Heveanch aiws:

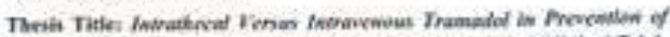

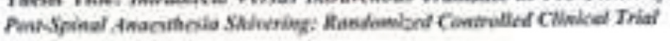
Devar Dr:

Think you ker geod letiet is $(23 / 2020)$ so sok for the ethical

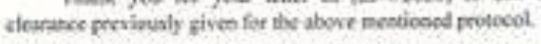

We are plessed io cenfirm that the abere meativoed pratotel of oneiti way

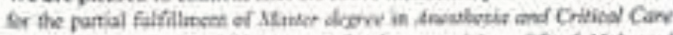

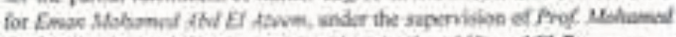

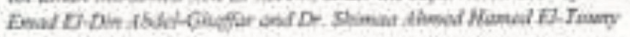

The properal of the above mentined pretecel azs resiened by the fill

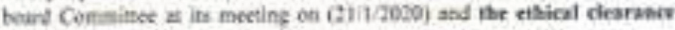
was given for chat praponal.

Fown sinciridfy.

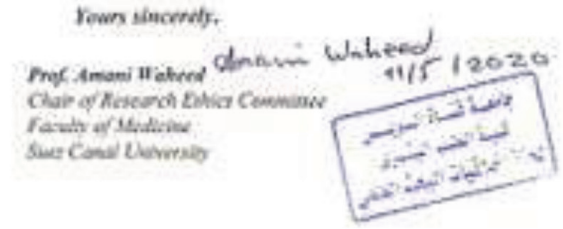

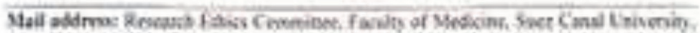

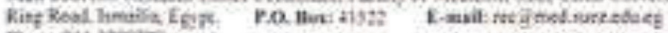

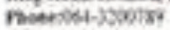

\section{Abstract 49 Figure 1}

Abstract 49 Table 1 Patient characteristics of the two groups

\begin{tabular}{|c|c|c|c|}
\hline \multirow{2}{*}{ Variahles } & \multicolumn{2}{|c|}{ Type of tramadol sdivinhitration } & \multirow[b]{2}{*}{ prales } \\
\hline & $\begin{array}{c}\text { hotravesous } \\
\text { tramedel }(n-4)\end{array}$ & $\begin{array}{l}\text { Iatratiocal } \\
\text { Ifamadel (n-49) }\end{array}$ & \\
\hline Age (years), meas S SD & $45.50=11.53$ & $48.07 \pm 13.33$ & 0.102 \\
\hline \multicolumn{4}{|l|}{ Groder, a (46) } \\
\hline Male & $24(565)$ & $26(60, A)$ & \multirow{2}{*}{$060^{\circ}$} \\
\hline Fexale & $19(43,5)$ & $17(39.6)$ & \\
\hline Weight (Ka), mataa = SD & $739=123$ & $79.3=11.6$ & $0.05 *$ \\
\hline Heblat (a), mavas = SD & $165.1=1.9$ & $\$ 69.5 \pm 1.0\}$ & $0.06=$ \\
\hline BMII (hg/ im's mesin z SD & $2937 \pm+1.4$ & $30.53 \geq 5.37$ & 0.267 \\
\hline \multicolumn{4}{|l|}{ Ineidenet of thiverins: } \\
\hline \multirow[t]{2}{*}{ Abreat $\mathrm{N}(\mathrm{SS})$} & $35(81.4)$ & $44(95.4)$ & \multirow{3}{*}{$0.647 *$} \\
\hline & & & \\
\hline presest N (b) & $8(18.6)$ & $2(4.6)$ & \\
\hline \multicolumn{4}{|c|}{ 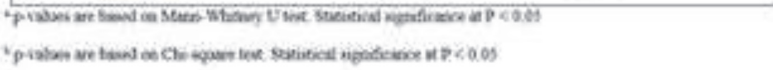 } \\
\hline 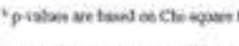 & 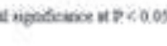 & & \\
\hline 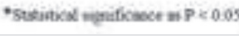 & & & \\
\hline
\end{tabular}

Abstract 49 Table 2 Post-operative complications among the two groups

\begin{tabular}{|c|c|c|c|}
\hline \multirow[b]{2}{*}{ Variables } & \multicolumn{2}{|c|}{ T)pe of tramadal adminhitration } & \multirow[b]{2}{*}{ p-value } \\
\hline & $\begin{array}{c}\text { Latranrauas } \\
\text { tranesdel }\left(a^{-49}\right)\end{array}$ & $\begin{array}{c}\text { Intrathecal } \\
\text { tramadol }(n-43)\end{array}$ & \\
\hline \multicolumn{4}{|l|}{ Nauses } \\
\hline Forient & $12(27.9)$ & $4(93)$ & \multirow{2}{*}{$20.026^{2}+$} \\
\hline Abrent & $31(72.1)$ & 39007 & \\
\hline \multicolumn{4}{|l|}{ Vomition } \\
\hline fresent & $4(9.3)$ & $2(4.6)$ & \multirow{2}{*}{0.396} \\
\hline Ableta & $39(90.7)$ & $41,954\}$ & \\
\hline \multicolumn{4}{|c|}{ Hspolearbe } \\
\hline Puesen & $2(4.6)$ & $3(7)$ & \multirow{2}{*}{$0.21 \%$} \\
\hline Abotena & $41(8,4)$ & $40(93)$ & \\
\hline
\end{tabular}

Results Shivering was observed in $8(18.6 \%)$ patients in intravenous group compared to $2(4.6 \%)$ patients in intrathecal group (P-value of 0.047).

Postoperative nausea occurred in $12(27.9 \%)$ patients in intravenous group versus $4(9.3 \%)$ patients in intrathecal group with a p-value of 0.026 .

Conclusions Intra-thecal tramadol prevents post-spinal shivering without complications.

\section{THE USE OF SHORT-ACTING SPINAL ANAESTHESIA TO IMPROVE RECOVERY IN COLORECTAL SURGERY}

S Dean*, M Gorecha, M Pais, K Dasgupta, Z Aslam. George Eliot Hospital NHS Trust, Nuneaton, UK

\subsection{6/rapm-2021-ESRA.50}

Background and Aims Colorectal surgery has evolved to laparoscopic procedures with long-acting spinal anaesthesia and intrathecal opiates over epidural anaesthesia [1]. This has become the standard for enhanced recovery programmes across the world with good post-op analgesia [2]. However, due to the longer duration of action of spinal bupivacaine, there are increased risks of hypotension, delayed mobilisation and urinary retention [3]. This case-series presents the use of a short-acting prilocaine spinal anaesthetic in combination with intrathecal opiates and abdominal wall blocks, which successfully provide sufficient analgesia whilst promoting enhanced recovery in laparoscopic colorectal surgery.

Methods Twenty patients undergoing laparoscopic colorectal surgery were included. These patients received $3 \mathrm{ml}$ of intrathecal 2\% hyperbaric prilocaine combined with 500micrograms of diamorphine combined with rectus sheath and transversus

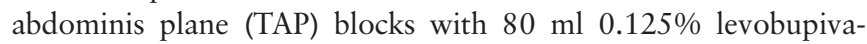
caine as part of the enhanced recovery protocol.

Results Intraoperatively, patients were observed to be more haemodynamically stable and post-operatively, all patients' blood-pressure readings had returned to pre-operative levels without the need for any vasopressor support. In the recovery area they were comfortable, with pain scores of 0 , and were able to sit-up and ambulate faster without any vasovagal episodes.

Conclusions We have successfully shown that the action of prilocaine can be prolonged by the addition of multimodal analgesia including long-acting interfascial plane blocks. We suggest that it's use offers increased haemodynamic stability and earlier patient mobilisation post-operatively. This modified technique allows excellent long duration analgesia 